\title{
ISOMORPHISMS BETWEEN INVERSE SEMIGROUPS OF INJECTIVE TRANSFORMATIONS
}

\author{
BRIDGET BOS BAIRD
}

(Received 10 February 1976)

\begin{abstract}
We introduce a class of inverse semigroups of injective transformations and our main result concerns isomorphisms between two such semigroups. This result is then applied to semigroups of homeomorphisms between closed subsets of a $T_{1}$ topological space, semigroups of homeomorphisms between compact subsets of $\boldsymbol{k}$-spaces and semigroups of isomorphisms between subsemilattices of semilattices. In the first two cases it is shown that the two inverse semigroups under consideration are isomorphic if and only if the corresponding topological spaces are homeomorphic. In the latter case, the two inverse semigroups are isomorphic if and only if the semilattices are either isomorphic or are dual isomorphic infinite chains.
\end{abstract}

\section{Introduction}

An element $b$ in a semigroup $S$ is an inverse of an element $a$ in $S$ if $a b a=a$ and $b a b=b$. In general, an element may have more than one inverse. Those semigroups in which each element has precisely one inverse are referred to as inverse semigroups. The canonical example of an inverse semigroup is the semigroup, under composition, of all injective partial transformations on a set. This inverse semigroup is denoted by $\mathscr{I}_{X}$ and is referred to as the symmetric inverse semigroup on the set $X$. Preston (1954) and Vagner (1952) have shown that every inverse semigroup $S$ can be embedded in $\oiint_{s}$. One may consult Clifford and Preston (1961) for a discussion of inverse semigroups in general and the symmetric inverse semigroup in particular.

Preston (1973) has suggested various natural ways in which one can extend the notion of the symmetric inverse semigroup on a set $X$. One can provide $X$ with some sort of structure and then take all those injective partial

The results presented in this paper are taken from the author's doctoral dissertation written at the State University of New York at Buffalo under the direction of K. D. Magill, Jr. 
maps on $X$ which are compatible in some sense with that structure. For example, one can take $X$ to be a semigroup and the inverse semigroup to consist of all isomorphisms between subsemigroups of $X$. (The empty set is regarded as a subsemigroup of $X$.) For another example, take $X$ to be a topological space and the inverse semigroup to consist of all homeomorphisms between closed subsets of $X$. We denote this latter semigroup by $\mathscr{I}_{F}(X)$. The results in this paper are, for the most part, a consequence of our investigations of the semigroups $\mathscr{I}_{F}(X)$ where $X$ is any $T_{1}$ space. In particular we wanted to determine if the $T_{1}$ spaces $X$ and $Y$ must be homeomorphic whenever the semigroups $\mathscr{I}_{F}(X)$ and $\mathscr{I}_{F}(Y)$ are isomorphic (it is a straightforward matter to show that the converse is true). They must be, and what we do is to prove a rather general theorem which can be applied to obtain this result not only for $\mathscr{I}_{F}(X)$ but for other inverse semigroups on topological spaces as well. In fact, the result is general enough to apply to the inverse semigroup of all isomorphisms between subsemilattices of a semilattice (that is, a commutative semigroup all of whose elements are idempotent). In this case, two such inverse semigroups are isomorphic if and only if the semilattices are either isomorphic or are dual isomorphic infinite chains.

The author would like to thank the referee for pointing out an error in the original version of this paper.

\section{Definition of $\mathscr{I}(X)$ and examples}

Definition 2.1. Let $X$ be a set and $\mathscr{F}$ a collection of subsets of $X$ such that if $F$ and $F^{\prime}$ belong to $\mathscr{F}$ then $F \cap F^{\prime}$ belongs to $\mathscr{F}$. Then $\mathscr{I}(X)$ will, in general, denote any collection of injective maps whose domains and ranges are members of $\mathscr{F}$ satisfying the following conditions:

1) For any $F \in \mathscr{F}$, the identity map on $F$, denoted $i_{F}$, belongs to $\mathscr{I}(X)$.

2) If $f$ belongs to $\mathscr{I}(X)$ then the inverse mapping $f^{-1}$ of $f$ also belongs to $\mathscr{I}(X)$.

3) $\mathscr{F}(X)$ is closed with respect to composition of functions.

It can easily be shown that $\mathscr{I}(X)$ is an inverse semigroup where the inverse of the map $f$ is just $f^{-1}$. Idempotents in $\mathscr{T}(X)$ are of the form $i_{F}$ for $F \in \mathscr{F}$. To emphasize association with $X$ the set $\mathscr{F}$ will sometimes be written $\mathscr{F}(X)$. Examples of various $\mathscr{F}(X)$ are given below, together with their own special designations. Proofs that these examples satisfy the requirements of Definition 2.1 are straightforward.

1) $X$ is any nonempty topological space, $\mathscr{F}=\{F: F$ is a closed subset of $X$ \}, and $\mathscr{I}_{F}(X)=\{f$ : $\operatorname{dom} f$ (the domain of $f$ ) is closed, $\operatorname{ran} f$ (the range of $f$ ) is closed, and $f$ is a homeomorphism\}. 
2) $X$ is any nonempty topological space, $\mathscr{F}=\{K: K$ is a closed compact subset of $X\}$, and $\mathscr{I}_{K}(X)=\{f: \operatorname{dom} f \in \mathscr{F}, \quad$ ran $f \in \mathscr{F}$, and $f$ is a homeomorphism\}.

3) $X$ is any semigroup, $\mathscr{F}=\{S: S$ is a subsemigroup of $X\}$, and $\mathscr{I}_{s}(X)=\{f: \operatorname{dom} f$ is a subsemigroup of $X$, ran $f$ is a subsemigroup of $X$, and $f$ is an isomorphism\}.

DEFINITION 2.2. The inverse semigroup $\mathscr{F}(X)$ (with associated set $\mathscr{F}(X)$ ) will be called maximal if $\mathscr{I}(X)$ contains all maps $f$ which satisfy the following conditions:

1) $f$ is an injective map

2) $\operatorname{dom} f \in \mathscr{F}(X)$ and $\operatorname{ran} f \in \mathscr{F}(X)$

3) if $F \in \mathscr{F}(X)$ then $f(F) \in \mathscr{F}(X)$ and $f^{-1}(F) \in \mathscr{F}(X)$.

\section{Isomorphism theorems}

THEOREM 3.1. Let $X$ and $Y$ be nonempty sets such that $\{x\} \in \mathscr{F}(X)$ for every $x \in X$ and $\{y\} \in \mathscr{F}(Y)$ for every $y \in Y$. Let $\varphi$ be an isomorphism from $\mathscr{I}(X)$ onto $\mathscr{I}(Y)$. Then there exists a unique bijection $h$ from $X$ onto $Y$ such that:

1) $h(F) \in \mathscr{F}(Y)$ for all $F \in \mathscr{F}(X), h^{-1}(H) \in \mathscr{F}(X)$ for all $H \in \mathscr{F}(Y)$

2) $\varphi(f)=h \circ f \circ h^{-1}$ for all $f \in \mathscr{I}(X)$.

Conversely, if $\mathscr{I}(X)$ and $\mathscr{I}(Y)$ are maximal then all isomorphisms from $\mathscr{I}(X)$ onto $\mathscr{I}(Y)$ are obtained in exactly this way.

Proof. Suppose $\varphi$ is an isomorphism from $\mathscr{I}(X)$ on to $\mathscr{I}(Y)$. Denote by $\langle x, z\rangle$ the map $f$ such that $\operatorname{dom} f=\{x\}$ and $f(x)=z$. Let $x \in X$. Since $\{x\} \in \mathscr{F}(X)$ for every $x \in X,\langle x, x\rangle$ belongs to $\mathscr{I}(X)$. The homomorphic image of an idempotent is an idempotent and so $\varphi\langle x, x\rangle=i_{V}$ for some $V \in \mathscr{F}(Y)$. Suppose $V=\varnothing$. Then $\varphi^{-1}\left(i_{V}\right)$ is the zero for $\mathscr{I}(X)$. This means that $\langle x, x\rangle \circ\langle z, z\rangle=\langle x, x\rangle$ for every $z \in X$. Hence $X=\{x\}$. Now $i_{\varnothing} \notin \mathscr{I}(X)$ since $\langle x, x\rangle$ is the zero for $\mathscr{F}(X)$. Hence $\mathscr{I}(X)=\{\langle x, x\rangle\}$. The map $\varphi$ is an isomorphism and so $|\mathscr{I}(Y)|=1$. Since $Y$ is nonempty we have that $\langle y, y\rangle \in$ $\mathscr{I}(Y)$ for some $y \in Y$. But $i_{\varnothing} \in \mathscr{I}(Y)$ also. Hence $|\mathscr{I}(Y)| \geqq 2$ which is a contradiction. This means that $V \neq \varnothing$. Now let $y \in V$. Then $\varphi^{-1}\langle y, y\rangle$ is an idempotent. Suppose $\varphi^{-1}\langle y, y\rangle=i_{U}$. Then

$$
\varphi\left(i_{U \cap\{x\}}\right)=\varphi\left(i_{U} \circ\langle x, x\rangle\right)=\varphi\left(i_{U}\right) \circ \varphi\langle x, x\rangle=\langle y, y\rangle \circ i_{V}=\langle y, y\rangle=\varphi\left(i_{U}\right) .
$$

Since $\varphi$ is injective this means that $U \cap\{x\}=U$. Now $U \neq \varnothing$ by the same reasoning as above. Hence $U=\{x\}$. Therefore $\varphi\langle x, x\rangle=\langle y, y\rangle$. Set $h(x)=y$. Then $\varphi\langle x, x\rangle=\langle h(x), h(x)\rangle$. Since $\varphi$ is an isomorphism we have that $h$ is an 
injective map. If $y \in Y$, then the same reasoning as above shows that $\varphi^{-1}\langle y, y\rangle=\langle x, x\rangle$ for some $x \in X$. Clearly $h(x)=y$. This shows that $h$ is a bijection from $X$ onto $Y$.

Now it will be shown that $\varphi\left(i_{U}\right)=i_{r(U)}$. Let $\varphi\left(i_{U}\right)=i_{V}$ and suppose $x \in U$. Then $i_{U} \circ\langle x, x\rangle=\langle x, x\rangle$ and

$$
\langle h(x), h(x)\rangle=\varphi\langle x, x\rangle=\varphi\left(i_{U} \circ\langle x, x\rangle\right)=\varphi\left(i_{U}\right) \circ \varphi\langle x, x\rangle=i_{V} \circ\langle h(x), h(x)\rangle .
$$

Therefore $h(x) \in V$ and so $h(U) \subseteq V$. Conversely, if $y \in V$ then $y=h(x)$ for some $x \in X$. Now

$$
\begin{aligned}
\langle x, x\rangle & =\varphi^{-1}\langle h(x), h(x)\rangle=\varphi^{-1}\left(i_{\vee} \circ\langle h(x), h(x)\rangle\right) \\
& =\varphi^{-i}\left(i_{\vee}\right) \circ \varphi^{-1}\langle h(x), h(x)\rangle=i_{U} \circ\langle x, x\rangle .
\end{aligned}
$$

Hence $x \in U$ and so $V \subseteq h(U)$. Thus $V=h(U)$ and $\varphi\left(i_{U}\right)=i_{h(U)}$. Since $\varphi\left(i_{U}\right) \in \mathscr{I}(Y)$ this shows that $h(U) \in \mathscr{F}(Y)$. Conversely, if $H \in \mathscr{F}(Y)$ then $\varphi^{-1}\left(i_{H}\right) \in \mathscr{I}(X)$. But if $\varphi^{-1}\left(i_{H}\right)=i_{U}$ then $h(U)=H$ and since $h$ is a bijection this means that $h^{-1}(H)=U$ and hence $h^{-1}(H) \in \mathscr{F}(X)$. Thus part 1) of the theorem has been shown.

Suppose $f \in \mathscr{I}(X)$. We will show that $\varphi(f)=h \circ f \circ h^{-1}$. Now

$$
\begin{aligned}
& \operatorname{dom} \varphi(f)=\operatorname{dom}\left((\varphi(f))^{-1} \circ \varphi(f)\right)=\operatorname{dom}\left(\varphi\left(f^{-1}\right) \circ \varphi(f)\right) \\
& \quad=\operatorname{dom} \varphi\left(f^{-1} \circ f\right)=\operatorname{dom} \varphi\left(i_{\operatorname{dom} f}\right)=\operatorname{dom}\left(i_{h(\operatorname{dom} f)}\right)=h(\operatorname{dom} f) .
\end{aligned}
$$

Since $h$ is a bijection from $X$ onto $Y, \operatorname{dom}\left(h \circ f \circ h^{-1}\right)=h(\operatorname{dom} f)$ also. Now let $y \in \operatorname{dom} \varphi(f)$. Then $y=h(x)$ where $x \in \operatorname{dom} f$ and $\left(h \circ f \circ h^{-1}\right)(y)=$ $h(f(x))$. Now

$$
\begin{aligned}
\langle h(x), \varphi(f)(h(x))\rangle & =\varphi(f) \circ\langle h(x), h(x)\rangle=\varphi(f) \circ \varphi\langle x, x\rangle \\
& =\varphi(f \circ\langle x, x\rangle)=\varphi\langle x, f(x)\rangle .
\end{aligned}
$$

Thus $\varphi\langle x, f(x)\rangle$ maps $h(x)$ onto $\varphi(f)(h(x))$. We also have that

$$
\begin{aligned}
\operatorname{ran} \varphi\langle x, f(x,) & =\operatorname{dom}(\varphi\langle x, f(x)\rangle)^{-1}=\operatorname{dom} \varphi\left(\langle x, f(x)\rangle^{-1}\right)=\operatorname{dom} \varphi\langle f(x), x\rangle \\
& =h(\operatorname{dom}\langle f(x), x\rangle)=\{h(f(x))\} .
\end{aligned}
$$

Hence $\varphi(f)(h(x))=h(f(x))$ and so $\varphi(f)=h \circ f \circ h^{-1}$. Thus 2) has been established.

To complete the first part of the proof it must be shown that $h$ is unique. Therefore suppose $g$ is a bijection from $X$ onto $Y$ satisfying 1 ) and 2). Let $x \in X$. Because $g$ satisfies 2) we get that $\varphi\langle x, x\rangle=g \circ\langle x, x\rangle \circ g^{-1}$. But $\varphi\langle x, x\rangle=\langle h(x), h(x)\rangle$. Hence $g^{-1}(h(x))=x$ and $g(x)=h(x)$. Thus $g=h$ and $h$ is unique. 
To show the converse of the theorem suppose that $\mathscr{I}(X)$ and $\mathscr{I}(Y)$ are maximal and $h$ is a bijection from $X$ onto $Y$ satisfying 1). For $f \in \mathscr{I}(X)$ define $\varphi(f)=h \circ f \circ h^{-1}$. It must be shown that $\varphi$ is an isomorphism from $\mathscr{I}(X)$ onto $\mathscr{I}(Y)$. First we show that $\varphi(f) \in \mathscr{I}(Y)$. We have $\operatorname{dom} \varphi(f)=$ $h(\operatorname{dom} f)$. But $h(\operatorname{dom} f) \in \mathscr{F}(Y)$ by 1$)$ since $\operatorname{dom} f \in \mathscr{F}(X)$. Now $\operatorname{ran} \varphi(f)=$ $\operatorname{dom}\left((\varphi(f))^{-1}\right)=\operatorname{dom}\left(\left(h \circ f \circ h^{-1}\right)^{-1}\right)=h(\operatorname{ran} f)$. Again, by 1$), \quad \operatorname{ran} \varphi(f) \in$ $\mathscr{F}(Y)$. Clearly $\varphi(f)$ is an injective map. Now let $F \in \mathscr{F}(Y)$ and set $\operatorname{dom} \varphi(f)=V$. Then

$$
\varphi(f)(F)=\varphi(f)(F \cap V)=\left(h \circ f \circ h^{-1}\right)(F \cap V) .
$$

Now $F \cap V \in \mathscr{F}(Y)$ and so, by requirement 1$), h^{-1}(F \cap V) \in \mathscr{F}(X)$. But then $\left(f \circ h^{-1}\right)(F \cap V) \in \mathscr{F}(X)$ also and hence $\left(h \circ f \circ h^{-1}\right)(F \cap V) \in \mathscr{F}(Y)$. Thus $\varphi(f)(F \cap V) \in \mathscr{F}(Y)$. Likewise $(\varphi(f))^{-1}(F) \in \mathscr{F}(Y)$ also. Since $\mathscr{I}(Y)$ is maximal we have that $\varphi(f) \in \mathscr{I}(Y)$. Thus $\varphi$ maps $\mathscr{I}(X)$ into $\mathscr{I}(Y)$. If $g \in \mathscr{I}(Y)$ then it can easily be shown that the map $h^{-1} \circ g \circ h$ belongs to $\mathscr{I}(X)$ (using the maximality of $\mathscr{I}(X)$ and the fact that $h$ satisfies 1$)$ ). Clearly $\varphi\left(h^{-1} \circ g \circ h\right)=g$ and so $\varphi$ maps $\mathscr{I}(X)$ onto $\mathscr{I}(Y)$. Since $h$ is injective $\varphi$ must also be injective. To complete the proof it only must be shown that $\varphi$ is a homomorphism. Let $f, g \in \mathscr{I}(X)$. Then

$$
\begin{aligned}
\varphi(f \circ g) & =h \circ(f \circ g) \circ h^{-1}=h \circ f \circ i_{X} \circ g \circ h^{-1} \\
& =h \circ f \circ h^{-1} \circ h \circ g \circ h^{-1}=\varphi(f) \circ \varphi(g) .
\end{aligned}
$$

This shows that $\varphi$ is an isomorphism from $\mathscr{I}(X)$ onto $\mathscr{I}(Y)$.

This theorem will now be used to prove isomorphism theorems for $\mathscr{I}_{F}(X)$ where $X$ is a $T_{1}$ space, for $\mathscr{I}_{K}(X)$ where $X$ is a $k$-space, and for $\mathscr{I}_{s}(X)$ where $X$ is a semilattice.

Corollary 3.2. Suppose $X$ and $Y$ are nonempty topological $T_{1}$ spaces. For each isomorphism $\varphi$ from $\mathscr{I}_{F}(X)$ onto $\mathscr{I}_{F}(Y)$ there exists a unique homeomorphism $h$ from $X$ onto $Y$ such that $\varphi(f)=h \circ f \circ h^{-1}$ for each $f \in \mathscr{I}_{F}(X)$. Conversely, if $h$ is a homeomorphism from $X$ onto $Y$, then the mapping $\varphi$, defined by $\varphi(f)=h \circ f \circ h^{-1}$ for each $f \in \mathscr{I}_{F}(X)$, is an isomorphism from $\Phi_{F}(X)$ onto $\mathscr{I}_{F}(Y)$.

Proof. Since $X$ and $Y$ are $T_{1}$ spaces, they satisfy the conditions of the theorem. If $\varphi$ is an isomorphism from $\mathscr{I}_{F}(X)$ onto $\mathscr{I}_{F}(Y)$ then there is a unique bijection $h$ from $X$ onto $Y$ satisfying 1) and 2). This just means that $h$ is a homeomorphism.

It can easily be shown that the sets $\mathscr{I}_{F}(X)$ and $\mathscr{\Phi}_{F}(Y)$ are maximal. Thus the converse also follows from the last theorem. 
A topological $T_{2}$ space $X$ is a $k$-space if a set $A \subseteq X$ is closed if and only if the intersection $A \cap K$ is closed for every compact set $K \subseteq X$. The class of $k$-spaces includes all locally compact $T_{2}$ spaces as well as all first countable $T_{2}$ spaces (Dugundji (1966)). A useful advantage in working with $k$-spaces is that in order to show that a bijection $h$ from one such space $X$ onto another such space $Y$ is a homeomorphism, it is sufficient to show that both $h$ and $h^{-1}$ carry compact sets onto compact sets. With this additional fact in mind the next result follows in much the same manner as the last corollary.

Corollary 3.3. Suppose $X$ and $Y$ are nonempty $T_{1}$ spaces. For each homeomorphism $h$ from $X$ onto $Y$, the mapping $\varphi$, defined by $\varphi(f)=h \circ f \circ h^{-1}$ for each $f \in \mathscr{I}_{K}(X)$, is an isomorphism from $\mathscr{I}_{K}(X)$ onto $\mathscr{I}_{K}(Y)$. If $X$ and $Y$ are $k$-spaces then the converse holds in the sense that for each isomorphism $\varphi$ from $\mathscr{I}_{K}(X)$ onto $\mathscr{I}_{K}(Y)$, there exists a unique homeomorphism $h$ from $X$ onto $Y$ such that $\varphi(f)=h \circ f \circ h^{-1}$ for each $f \in \mathscr{I}_{K}(X)$.

Recall that a set $X$ is a semilattice if $X$ is a commutative semigroup and every element of $X$ is idempotent. Then $\mathscr{I}_{s}(X)$ will consist of all isomorphisms between subsemilattices of the semilattice $X$ (the empty set is considered to be a subsemilattice of $X$ ). A bijection $h$ from a semilattice $X$ onto a semilattice $Y$ is called a dual isomorphism if for every $a, b \in X, a b=a$ if and only if $h(a) h(b)=h(b)$.

THEOREM 3.4. Suppose $X$ and $Y$ are semilattices. If $\varphi$ is an isomorphism

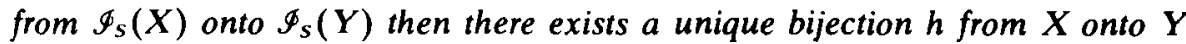
such that $\varphi(f)=h \circ f \circ h^{-1}$ for every $f \in \mathscr{I}_{S}(X)$. Either $h$ is an isomorphism or $X$ and $Y$ are chains and $h$ is a dual isomorphism. Conversely, if $h$ is an isomorphism from $X$ onto $Y$ or both $X$ and $Y$ are chains and $h$ is a dual isomorphism then the map $\varphi$, defined by $\varphi(f)=h \circ f \circ h^{-1}$ for each $f \in \mathscr{I}_{s}(X)$, is an isomorphism from $\mathscr{I}_{S}(X)$ onto $\mathscr{I}_{S}(Y)$.

Proof. Suppose $\varphi$ is an isomorphism from $\mathscr{I}_{s}(X)$ onto $\mathscr{I}_{s}(Y)$. If $X=\varnothing$ then $\left|\mathscr{I}_{S}(X)\right|=1$ and $\left|\mathscr{I}_{S}(Y)\right|=1$ also and $Y=\varnothing$. Hence suppose $X \neq \varnothing$ and $Y \neq \varnothing$. Since $X$ and $Y$ are semilattices we have that $\{x\}$ is a subsemilattice of $X$ for every $x \in X$ and $\{y\}$ is a subsemilattice of $Y$ for every $y \in Y$. We now apply Theorem 3.1 to get a unique bijection $h$ from $X$ onto $Y$ such that $\varphi(f)=h \circ f \circ h^{-1}$ for $f \in \mathscr{I}_{s}(X)$. This means that $K$ is a subsemilatice of $X$ if and only if $h(K)$ is a subsemilattice of $Y$. First suppose that there exist $x, y \in X$ such that $x y \neq x$ and $x y \neq y$. Then $K=\{x, y, x y\}$ is a subsemilattice of $X$ and so $h(K)=\{h(x), h(y), h(x y)\}$ is a subsemilattice of $Y$. Thus $h(x) h(y) \in h(K)$. If $h(x) h(y)=h(x)$ or $h(x) h(y)=h(y)$ then $\{h(x), h(y)\}$ is a subsemilattice of $Y$ and so $\{x, y\}$ is a subsemilattice of $X$ which is a 
contradiction. Thus $h(x) h(y)=h(x y)$. Now let $a$ and $b$ be distinct elements of $X$. If $a b \neq a$ and $a b \neq b$ then $h(a b)=h(a) h(b)$ by the above reasoning. Hence suppose $a b=a$. Recall that we are assuming that there exist $x, y \in X$ such that $x y \neq x$ and $x y \neq y$ and so we have that $h(x) h(y)=h(x y)$, Define a map $f \in \mathscr{I}_{s}(X)$ from $\{x y, x\}$ onto $\{a, b\}$ by $f(x y)=a, f(x)=b$. Then $f$ is an isomorphism. Thus $\varphi(f)$ is an isomorphism which maps $\{h(x y), h(x)\}$ onto $\{h(a), h(b)\}$ and so

$$
\varphi(f)(h(x y)) \varphi(f)(h(x))=\varphi(f)(h(x y) h(x)) .
$$

Now

$$
h(x y) h(x)=h(x) h(y) h(x)=h(y) h(x)=h(x y)
$$

and so

$$
\begin{aligned}
h(a) & =h(f(x y))=\left(h \circ f \circ h^{-1}\right)(h(x y))=\varphi(f)(h(x y)) \\
& =\varphi(f)(h(x y) h(x))=\varphi(f)(h(x y)) \varphi(f)(h(x)) \\
& =h(f(x y)) h(f(x))=h(a) h(b) .
\end{aligned}
$$

Thus $h(a) h(b)=h(a)=h(a b)$ and so $h$ is an isomorphism from $X$ onto $Y$. Now suppose there do not exist elements $x, y \in X$ such that $x y \neq x$ and $x y \neq y$. Then $X$ is just a chain. If $x, y \in X$ with $x y=x$ and $h(x) h(y)=h(x)$ then by the same reasoning as before we have that $h(a) h(b)=h(a b)$ for all $a, b \in X$ (define an isomorphism $f$ from $\{x, y\}$ onto $\{a, b\}$ ). Thus $h$ is an isomorphism. Now suppose $X$ is a chain, $x$ and $y$ belong to $X, x y=x$ but $h(x) h(y) \neq h(x)$. Since $\{h(x), h(y)\}$ is a subsemilattice we must have that $h(x) h(y)=h(y)$. Now every pair of elements in $Y$ forms a subsemilattice and so $Y$ is a chain also. Let $a, b \in X$ with $a b=a$. Then as before define a map $f$ from $\{x, y\}$ onto $\{a, b\}$ by $f(x)=a, f(y)=b$. Then $\varphi(f)$ is an isomorphism from $\{h(x), h(y)\}$ onto $\{h(a), h(b)\}$. Hence

$$
\begin{aligned}
h(a) h(b) & =\varphi(f)(h(x)) \varphi(f)(h(y))=\varphi(f)(h(x) h(y)) \\
& =\varphi(f)(h(y))=h(f(y))=h(b) .
\end{aligned}
$$

Now we have a bijection $h$ from the chain $X$ on to the chain $Y$ which satisfies the condition that if $a b=a$ then $h(a) h(b)=h(b)$. This means that $h$ is a dual isomorphism from $X$ onto $Y$.

For the converse of the theorem first suppose that $h$ is an isomorphism from $X$ onto $Y$. It is straightforward to show that $\varphi$, defined by $\varphi(f)=$ $h \circ f \circ h^{-1}$, is an isomorphism from $\mathscr{I}_{S}(X)$ onto $\mathscr{I}_{S}(Y)$. Now suppose $h$ is a dual isomorphism from the chain $X$ onto the chain $Y$. Define $\varphi(f)=$ $h \circ f \circ h^{-1}$. Then $\operatorname{dom} \varphi(f)=h(\operatorname{dom} f)$ which is a subsemilattice of $Y$. Also 
$\operatorname{ran} \varphi(f)$ is a subsemilattice of $Y$. Let $h(x), h(y) \in \operatorname{dom} \varphi(f)$. Then $x, y \in$ $\operatorname{dom} f$. Suppose $h(x) h(y)=h(x)$. Then $x y=y$ (h is a dual isomorphism). Hence $f(x) f(y)=f(y)$ and so $h(f(x)) h(f(y))=h(f(x))$. Now

$$
\begin{aligned}
\varphi(f)(h(x) h(y)) & =\varphi(f)(h(x))=h(f(x))=h(f(x)) h(f(y)) \\
& =\varphi(f)(h(x)) \varphi(f)(h(y)) .
\end{aligned}
$$

Thus $\varphi(f) \in \mathscr{I}_{s}(Y)$. It is straightforward to show that $\varphi$ is an isomorphism from $\mathscr{I}_{s}(X)$ onto $\mathscr{I}_{s}(Y)$.

Note that if two finite chains are dual isomorphic then they are isomorphic. This is clearly not so in the infinite case. The last theorem says that if $X$ and $Y$ are semilattices then $\mathscr{I}_{s}(X)$ and $\mathscr{I}_{S}(Y)$ are isomorphic if and only if $X$ and $Y$ are isomorphic or are dual isomorphic infinite chains.

Added in proof (3 November 1976). Corollary 3.2 also follows from a paper by W. J. Thron called 'Lattice-equivalence of topological spaces' in the Duke Mathematical Journal 29 (1962), 671-679.

\section{References}

A. H. Clifford and G. B. Preston (1961), 'The Algebraic Theory of Semigroups', vol. I (2nd. ed., Mathematical Surveys of the American Mathematical Society 7, Providence, R. I.).

J. Dugundji (1966), Topology (Allyn and Bacon, Inc., Boston).

G. B. Preston (1973), 'Inverse Semigroups: Some Open Questions' (Proceedings of a Symposium on Inverse Semigroups, Northern Illinois University).

G. B. Preston (1954), 'Representations of Inverse Semigroups', J. London Math. Soc. 29, 411-419.

V. V. Vagner (1952), 'Generalized Groups', Doklady Akad. Nauk SSSR (N.S.) 84, 1119-1122.

State University of New York at Buffalo, Amherst, New York 14226,

U.S.A. 